\title{
ENERGY EFFICIENT ROUTING PROTOCOL FOR WIRELESS BODY AREA NETWORKS (EER-W) USING COST COMPUTATION AND CONTROLLED HELLO PACKET BROADCAST
}

\author{
S. R Hemanth \\ Research Scholar, Department of Computer Science and Engineering, \\ VidyaVikas Institute of Engineering \& Technology Research Center \\ under Visvesvaraya Technological University, \\ Mysore - Bannur Road, Alanahally, Mysuru, Karnataka, India. \\ sr.hemanth@gmail.com \\ Dr.SanjayPande M B \\ Professor, Department of Computer Science and Engineering, \\ GM Institute of Technology, P.B No. 577006, 4th Main Rd, Davanagere, Karnataka 577002 \\ rakroop99@gmail.com
}

\begin{abstract}
An energy efficient routing mechanism in WBAN can reduce the energy usage in nodes for communication thereby improving the life time of the network. WBAN which is an autonomous network uses heterogeneous sensor nodes which are battery powered. These nodes are used for monitoring body parameters continuously. Routing operations are used to send the data from sensor nodes to sink which is the destination node. The nodes can also be used as intermediate nodes during routing operation. This routing operation consumes much of the energy in the network. Existing routing mechanisms using cost computing methodologies have adopted residual energy, distance metrics with number of hops as parameters. This paper adapts device type along with residual energy and distance with hop count for cost computation to find the next hop node used for forwarding the data. A novel mechanism of controlled Hello packet broadcasting reduces the control packets and improves energy efficiency in the network. The simulation outcome shows better performance compared to the modified new-attempt routing protocol (NEW-ATTEMPT) and forwarding packets randomly. The EER-W routing ensures the most optimal and feasible path is selected from the source to the coordinator or sink with dynamic updating of next hop and routing tables. This reduces the energy consumption and increases life time and network throughput.
\end{abstract}

Keywords: Wireless Body Area Network; Energy efficiency; Cost function; Routing; Throughput; Network Lifetime.

\section{Introduction}

The Changing life style is resulting in diverse health issues. Ageing population needs continuous medical monitoring and timely support. This may be a challenge for doctors and nurse. The need for primary health care services may increase by $25 \%$ by $2025[1][2]$.The projected demand and supply of health care workers is inadequate. Technology such as WBAN can help manage the above situation in a more reliable and flexible way to the convenience of the patient who is being monitored. This continuous monitoring using WBAN can serve as early warning systems which help detect life threatening events like high blood pressure, cardiac irregularities and save precious lives.

Effective energy management can extend the lifetime of these autonomous wireless networks [3],[4]. Design of routing protocol can influence the energy usage, lifetime and network stability. This has gained major attention among researchers [5], [6], [7]. Effectiveness of WBAN mainly depends on energy which is a key resource. Since batteries with limited capacity normally power a sensor node, conserving energy is an important issue for WBAN applications. 
Majority of the research in WBAN routing consider nodes with cost functions with different parameters to select the most feasible route among the available routes to the sink. In the popular literature cost computation is done either by the sink in a centralized fashion. However all the nodes frequently generating and transmitting hello packets results in more energy consumption impacting the network life time. The mechanisms proposed for routing table construction and frequent updating consumes more energy reducing network lifetime.

The proposed work EER-W uses efficient mechanisms for broadcasting Hello packets, neighbour table and routing table. EER-W stands apart from other similar protocols in managing hello packets and selecting the next hop node using both centralized and distributed approach. Along with the energy remaining, EER-W takes into consideration the type of device and location data of the nodes in neighbour table. This results in improvement of overall network performance in terms of network traffic, and packet delay. Simulations done using OMNET++ based Castalia simulator indicates improved performance of EER-W compared to NEW-ATTEMPT and Random Routing RR where no routing mechanisms are considered.

The specific contributions of work are

To develop an improvised mechanism for broadcasting Hello packets using Hello Message protocol .This reduces the overall network traffic and improves the residual energy of nodes .

To design a novel mechanism for discovery of neighbour nodes, constructing the next hop table and Routing table which can help reduce the traffic load in the network, consumption of energy by sensor nodes and improve reliability in BAN.

To propose Cost computation function which takes into account multiple parameters for dynamically deciding on the next hop node.

To set-up simulation environment for comparing the proposed protocol EER-W with NEW-ATTEMPT and random routing protocols.

The remaining sections in the paper are as follows. Section 2 has related works. Algorithms and procedures for EER-W considering energy efficiency is discussed in Section 3. Simulation results in Section 4 and 5 with analysis and comparison. .Paper is concluded in Section 6.

\section{Related Work}

K.N.Qureshi et al. [8]. had emphasized the need for reliable and energy efficient mechanisms to improve the performance of continuous health monitoring systems using Tree based Energy Efficient Routing scheme (EERNTS).Rapid changes in network topology can result in low reliability and increased energy consumption. EERNTS has addressed these issues by using tree based adaptive multihop routing approach. Based on the channel conditions, transmission power is chosen adaptively by reduced power consumption. Authors use CTP protocol as benchmark which uses fixed power transmission. The experimentation was done using the MicaZ simulator. The performance of the protocol was measured against important metrics like packet reception ratio (PRR), average hop Count and energy consumption. When compared to the bench mark protocol CBT, EER-NTS consumes $10 \%$ less energy and also delay is less by $30 \%$.EER-NTS uses relays as forwarder nodes to increase the network life time. However the number beacon signals sent by EER-NTS is very high consuming more energy. Efficient beacon transmission mechanism could be planned to reduce the beacon signals which in turn would reduce the energy consumption.

S. Ahmed et al. [9]. Proposed an energy efficient protocol LAEEBA -Link-Aware and Energy Efficient protocol for wireless Body Area networks. This protocol uses Cooperative mechanism among the nodes during routing .Here nodes learn together and work together to enhance the overall performance of the system. CO LAEEBA uses cost function to determine and select the route from a node to the sink. Nodes share distance and energy information with each other.This cooperative nature helps in gathering data frequently. There is an improvement in the packet throughput because of the cooperative nature of the algorithm. The authors have experimented based on the following performance criteria. Stability period, network lifetime and throughput. The algorithms have shown good performance for majority of the performance metrics. However the authors have taken into consideration only 8 nodes for the network setup and experimentation. This may not reflect the true performance and efficiency of the algorithm.

Abidi et al. [10]. proposed Cluster based Routing Protocol(CRPBA) for WBAN. This protocol was designed with an objective of minimizing energy utilization and increasing the network lifetime. Improvement in the above metrics can ensure long time monitoring without need for frequent battery recharge or change. This Protocol uses multi hop topology with cluster based mechanism. Data aggregation is performed at cluster heads and transmitted to the sink node. CRPBA was simulated with MATLAB using 24 bio medical sensors. However clustering can result in control message overhead for communication among nodes and also for choosing new cluster heads because of changes in the network topology. This results in higher energy consumption. No optimization techniques or co operation among nodes to improve network performance. 
Roy, C .Chowdhury et al. [11]. emphasized the need for taking into consideration the SAR(Specific Absorption Rate) limits. Authors have indicated that more energy consumption may give rise to heating of nodes. They also propose that using multi hop strategy because of efficient packet forwarding can have a major issue .Involving multiple nodes in forwarding packets results in more energy usage.Also the nodes heat up resulting in health hazards and tissue damages. Changes in the posture can also affect link quality and transmission power. The authors opinion that majority of the existing protocols using multi hop mechanism is not suitable for WBAN with a maximum of 15 to 20 nodes.A two hop cost based routing with relay nodes are used to improve on the packet delivery ratio. The protocol also looks into heat absorption, link and node reliability factors and tries to find the next hop avoiding routes which result in more temperature. The proposed protocol is compared against MPR and EEFR as benchmarks. The results reflect reduction in energy consumption with improved packet delivery ration for varying data rates. However limiting the protocol to 2-hop communication can have its own adverse effects. If a node which is not directly connected to the sink cannot reach it because of the 2-hop limitation, data will be lost. Even if the node tries to increase its transmission power to reach the sink the energy consumption will be more and also the node temperature may increase defeating the purpose. Authors have not addressed this crucial issue in their protocol along with mobility of nodes.

Smail et al. [12]. contributed to improving on the energy efficiency and network stability through ESR routing protocol for WBAN. This protocol selects links which are stable and uses less energy. The authors identify that majority of the routing protocols in WBAN ignore link stability.ESR uses cost function to select the nodes. A mathematical model for has been proposed for link stability resulting in more stable links being selected. This improves efficiency of packet transmission and throughput. However this protocol has been experimented taking only 8 nodes into consideration. Also no experimentations have been done considering the scalability factor in the network and its overall impact on the network lifetime.

V. Navya and P. Deepalakshmi [13]. Provided a mobility model which uses dynamic packet routing strategies to route packets. This routing mechanism uses mathematical modelling for priority and threshold based mechanisms which reduce energy and path loss also eliminating redundant packets. Existing routing protocols MATTEMPT and SIMPLE have been selected because of their relevance to the proposed protocol. This protocol uses placement strategy for nodes by placing the sink at the centre equidistant to each other. However this protocol which was built for mobility scenario has a dynamic topology with nodes continuously changing the positions. Network lifetime of this mobility model has not been explored comprehensively.

Javaid et al. [14] proposed iM-SIMPLE to resolve the energy consumption problem using integer linear programming. Mobility model with different postures were considered for minimum energy consumption and increased throughput. The protocol showed improved stability and throughput compared to similar protocols. However computational overhead increases data delivery in the network.

N. Kaur and S. Singh [15]. had contributed to improving on the network life time and energy efficiency with an optimized routing scheme E-OCER . The forwarder nodes are selected using cost function considering remaining node energy and link reliability. The routing mechanism has been optimized using Genetic algorithms. E-OCER uses multi-hop approach with reduced energy consumption and increased throughput. However more energy consumption of nodes near the sink can create additional energy losses. The packet drop rate as the network traffic increases is also a point of concern.

Y. Qu et al. [16]. had proposed an energy efficient routing protocol EER-NTP for transmitting the data reliably in a body area network. Normalization techniques are used to construct maximum benefit function using which the best next hop node is selected. The protocol achieves routing which is reliable and efficient also improves network efficiency and lifetime. However the protocol does not take into consideration reliability requirements when computing the weights which are important for achieving good performance in the network.

M. Anwar et al. [17]. have proposed ELR-W, an energy efficient routing protocol for WBAN. Authors have used crucial metrics like residual energy, efficiency of link, number of hop counts to determine path to the sink node. Experimentation of the above protocol was done using NS2 Simulator. Simulation results show improvement in throughput and lifetime of network with less energy consumption and packet loss.However use of Hello messages frequently results in high energy consumption. Higher packet drops are observed when the network traffic increases.

S. Singh, S. Negi, A. Uniyal [23]. had proposed an energy efficient routing protocol Modified new-Attempt routing protocol for WBAN. This protocol was designed with an objective of improving energy efficiency using cost function which computes the distance between the node and sink. The cost function uses residual energy and distance as metrics to decide on the path. The results demonstrate an improvement in energy efficiency .However the nodes broadcasting hello packets can drain the energy of sensor nodes. Cost function can consider other crucial parameters like type of sensor in WBAN to compute reliable path. 


\section{Devices in Body Area Network}

\subsection{Devices in a Body Area Network}

Human body physiological parameters like temperature, blood pressure, ECG, EEG etc can be collected and transmitted using Wireless Body Area networks. To provide comfort and mobility to the patients, the sensor nodes are wireless connected to each other using star topology. The sensor nodes are RFID's with limitations in processing and computation. It can only sense, transmit and receive data with limited processing. They can typically communicate within 3 meters range. The sink node which is also called as an actuator node is a transceiver with enhanced capabilities in terms of processing, memory and energy supply with power unit and processing unit .This can also hold and deliver medicine when needed. The sink node or body coordinator can transmit this data to monitoring stations using internet or cellular networks [18], [19].

Smart phones or PDA can be used as sink for user interaction and also data collection from sensor nodes. Both WSN and WBAN use sensor nodes for sensing data .But because of the differences in node type, coverage, topology accessibility, protocols designed for WSN and ad hoc networks cannot be used for WBAN.[20] , [21]

\subsection{Wireless Body Area Network Sensor}

\subsubsection{Wireless Body Area Network Sensor Nodes}

WBAN Sensor Nodes: Sensor nodes are miniature electronic devices which may be placed on the body or implanted within. These devices are equipped with processor, memory and transceiver. Based on the stimulus, sensors can sense and transmit the physiological parameters of human body.

\subsubsection{Actuators}

Hardware similar to sensor, but has additional hardware to hold medicines and can directly inject it into human body when needed. Actuators can act based on user instructions or data that is received from sensor nodes.

\subsubsection{Wireless Personal Device/Smart Phones}

Are devices which can receive data from sensors, actuators and send data to external devices like digital display or servers using gateways.[18]

\section{Proposed Work}

A novel mechanism has been introduced which provides information of when and to whom hello packets are to be broad casted. This result in less number of hello packets with reduced network traffic and energy consumption. Unlike NEW-ATTEMPT protocol only sinks and strategic nodes with high energy levels broad cast Hello packets. The proposed methodology delivers in 3 phases. 1) Novel Hello Message algorithm 2) Novel Neighbor table building algorithm and 3) Dynamic routing table building algorithm based on energy information and geographic location of node at that instance.

\subsection{Hello Message broadcasting}

Considering the above issues and keeping the energy efficiency and reliability in mind, a novel approach has been proposed with algorithms for discovery neighbor nodes, constructing the next hop table and Routing table. These mechanisms can help reduce the traffic load in the network, consumption of energy by sensor nodes and improve reliability in BAN.

This research work proposes an improvised mechanism for broadcasting Hello packets using Hello Message algorithm. The BAN nodes initially do not broad cast hello packets. BAN coordinator has information about all the sensor nodes. Initially the BAN coordinator will broad cast its Hello packets to neighboring BAN nodes. Assume Node $\mathrm{X}$ and $\mathrm{Y}$ are neighbour which receive these Hello packets. The neighbor information and routing tables are constructed by the nodes using the available information in the Hello packets, ex Node X and Node Y. After entering information into the tables Node $\mathrm{X}$ and Node $\mathrm{Y}$ will broadcast its own Hello packets. If Node $\mathrm{X}$ or Node Y does not receive Hello packets again within the specified interval it means that there is no direct link to the BAN Coordinator .In such case Node X or Node Y stops broadcasting Hello packets and also removes its routing table entries.

Devices used in BAN can be classified into 3 major classes based on the energy levels and frequency and ease with which we can recharge or replace the batteries. Our Model classifies the devices into 3 types. The sink which may be mobile phone or PDA which can be recharged regularly is Type 1.The nodes/relay nodes with easily replaceable batteries are considered as Type 2 and other BAN nodes including nodes which may be implanted with limited battery power and also which is difficult to replace the battery is considered as Type 3.Factors which play a major role in constructing the routing table are (i) Type of device i.e. Type 1,2 or 3 where a lower device type is preferred(ii)Neighbor with short distance to the sink (iii) Energy remaining in the node. These factors are major players in deciding in energy consumption and network traffic in the complete network as a whole. 


\subsection{Hello Message broadcasting}

The protocol builds on the assumption that Type 1 and Type 2 devices send Hello messages frequently. Different fields of Hello packet are depicted below. Node Y has the following fields in the Hello packet as shown below.

\begin{tabular}{|l|l|l|l|l|l|l|}
\hline IDENTDst & LOCDst & IDENTy & LOCj & DIST(y,„Dst) & REy & DTy \\
\hline
\end{tabular}

Figure 1: Hello Message packet format

The destination (Dst) can be Sink node, Relay Node or BAN node used as Next Hop. The different fields of the Hello packet contain the following information as shown in Table 1.

Table 1: Different fields of the Hello packet

\begin{tabular}{|l|l|}
\hline Notation & \multicolumn{1}{|c|}{ Meaning } \\
\hline IDENTDst & Destination device Identifier. \\
\hline LOCDst & Location of destination device \\
\hline IDENTy & Sender nodes Identifier \\
\hline DIST $(y$, ,Dst $)$ & Distance between sender Node $\mathrm{Y}$ to the destination \\
\hline REy & Remaining energy in Node $\mathrm{Y}$ \\
\hline DTy & Device Type \\
\hline
\end{tabular}

Node $\mathrm{Y}$ transmitting Hello packets received by Node $\mathrm{X}$ is stored in the neighbor table. Node $\mathrm{X}$ adds its own information to this data and broadcasts its Hello packets. Nodes can determine whether the sender is out of range or any issues with the link by considering the reception of new Hello packets within the defined interval. i.e. if the new Hello packet is not received within the time interval. In such case the neighbor table and routing table are updated.

$\operatorname{DIST}(\mathrm{y}, \mathrm{Dst})$ is calculated using equation 1:

$$
\operatorname{DIST}(\mathrm{y}, \text { Dst })=\sqrt{\left(\mathrm{M}_{\mathrm{y}}-\mathrm{M}_{\text {Dst }}\right)^{2}+\left(\mathrm{N}_{\mathrm{y}}-\mathrm{N}_{\text {Dst }}\right)^{2}}
$$

\subsection{Neighbor Table Construction Algorithm}

\subsubsection{Neighbor Table building algorithm}

Consider Node $i$ as the source, Node $\mathrm{j}$ as the intermediate node and destination node marked as Dst. Node $\mathrm{j}$ is located between Node $i$ and Dst. Node $i$ will have a Neighbor table with the following structure as shown in Figure(). The neighbor table contains the following information. IDENTDst is the identifier of destination device, LOCDst is the Location of destination device ,IDj which is ID of Neighbor ,LOCJ which is location of Neighbor, $\operatorname{DIST}(\mathrm{j}, \mathrm{Dst})$ which is distance to destination using $\mathrm{j}$ as neighbor node, DIST( $\mathrm{i}, \mathrm{j})$ which is distance from neighbor ,REji.e energy remaining in the neighboring node ,DTj which is the class to which node $\mathrm{j}$ belongs i.e Device Type, $\mathrm{Cj}$ which is cost of communication.

\begin{tabular}{|l|l|l|l|l|l|l|l|l|}
\hline IDENTDst & LOC $_{\text {Dst }}$ & IDj & LOCj & DIST(y,,Dst) & DIST(i,j) & REy & DTy & $C_{j}$ \\
\hline
\end{tabular}

The Hello Packet(hp) send by neighbor node is received by Node i .This received packet has information about the distance between the neighbor which is node $\mathrm{j}$ and Dst which is the destination i.e DIST(j,Dst)(hp).The algorithm will compare distance between the neighbor node $\mathrm{j}$ and the destination with that of direct link between node $\mathrm{i}$ to DIST(i, Dst) .New entry as a record is added by the algorithm if DIST(j,Dst) obtained through the hello packet is less compared to distance to the destination from node $\mathrm{i}$. This is represented by DIST(j,Dst) (hp ) < DIST(i, Dst).

To compute the distance between the two nodes i.e. node $i$, which is the source to node $j$, which is the neighbor node, the following equations can be used. The cost of communication to node $j$, can also be determined. Equations 2 and 3 are used to calculate the distance between the two nodes $i$ and $j$. The communication cost to node $\mathrm{j}$ is $\mathrm{Cj}$.

$$
\begin{aligned}
& \operatorname{DIST}(\mathrm{i}, \mathrm{j})=\sqrt{\left(\mathrm{M}_{\mathrm{i}}-\mathrm{M}_{\mathrm{j}}\right)^{2}+\left(\mathrm{N}_{\mathrm{i}}-\mathrm{N}_{\mathrm{j}}\right)^{2}} . \\
& C j=\frac{\left(\mathrm{DTj}^{2} * \mathrm{DIST}^{2}(\mathrm{i}, \mathrm{j})\right)}{\mathrm{RE}_{\mathrm{j}}} \ldots \ldots \ldots \ldots \ldots \ldots \ldots \ldots \ldots \ldots \ldots \ldots \ldots \ldots \ldots \ldots \ldots \ldots \ldots
\end{aligned}
$$




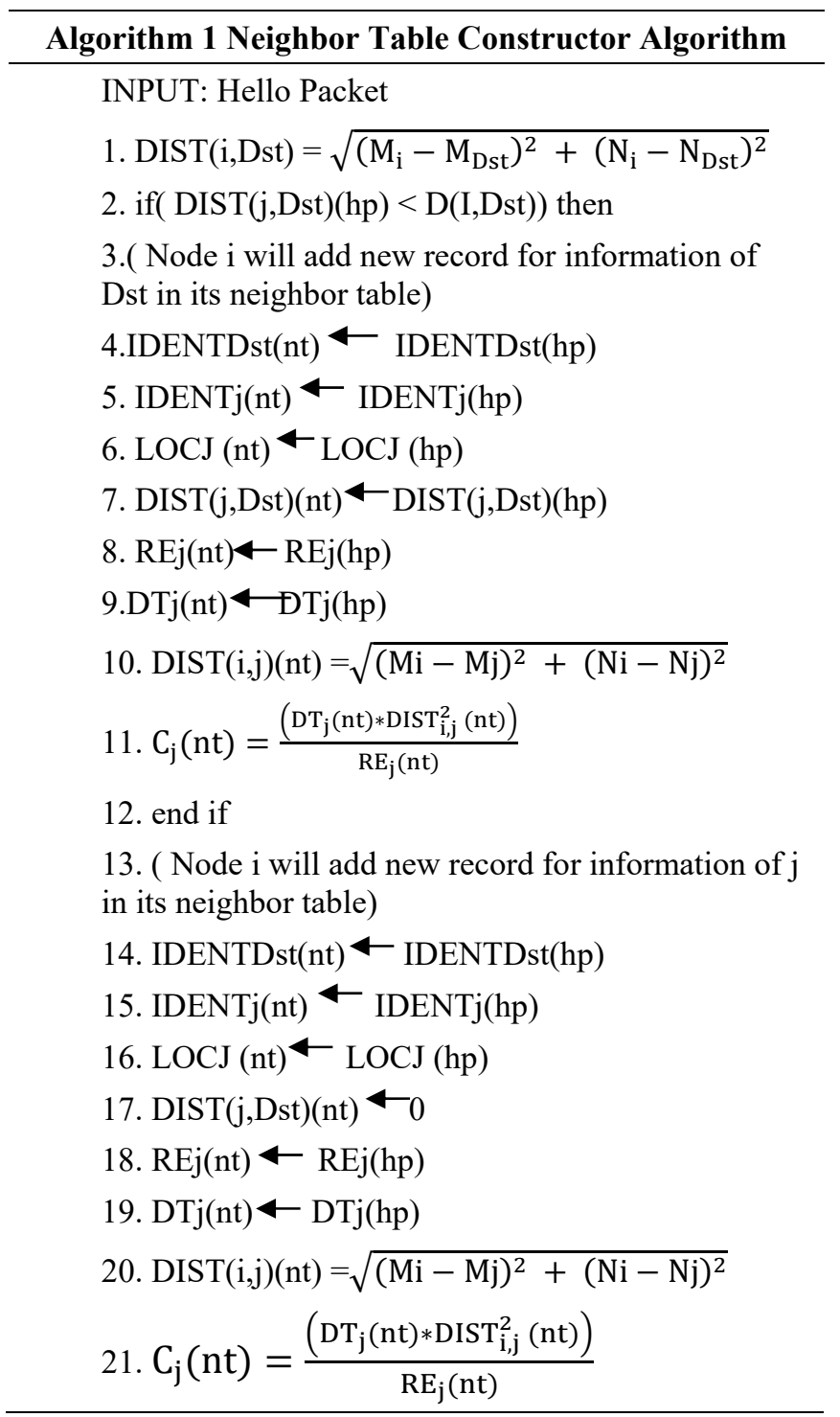

The free space transmission as an ideal scenario where only path loss is considered is as shown in equation 4. Here $\mathrm{Pt}(\mathrm{dBm})$ is transmission power of the signal and $\operatorname{Pr}(\mathrm{dBm})$ is received power. The gain of transmitter and receiver antenna is given by $\mathrm{Gt}$ and $\mathrm{Gr}$ respectively. $\lambda$ is wavelength and the distance between receiver and the transmitter is $\mathrm{R}$, as given in 4 .

$$
\operatorname{Pr}(\mathrm{dBm})=\operatorname{PtGtGr}\left(-\frac{\lambda}{4 \pi R}\right)^{2}
$$

In a real environment, propagation of wireless signals is influenced by various factors. Considering this and also multiple reflections results in change of path loss factor. Path loss power $\mathrm{L}(\mathrm{dB})$ is given equation 5 .

$$
\mathrm{L}=10 \lg \frac{P_{t}}{P_{r}} \text {. }
$$

RSSI which is the Received Signal Strength Indicator can be computed using the below equation. When we know the reference power P0 (dBm) at distance D0 (m) we can determine RSSI at distance D using 6.Here path loss exponent is represented by $\mathrm{n}$ and Gaussian random variable is $\mathrm{Xr}$.

$$
\mathrm{RSSI}=\mathrm{P} 0+10 \mathrm{n} \lg \left(\frac{D}{D_{0}}\right)+\mathrm{Xr}
$$

The neighbor table builder algorithm assumes that Hello packet transmitted by Node $\mathrm{j}$ is received by Nodei. In above algorithm, hp is Hello Packet , nt is Neighbor Table. Mi, Ni represents coordinates of node i .MDst and NDst represents coordinates if the destination. RSSI is used to calculate Mi and Ni of Node $\mathrm{i}$ as shown in the above equations. Different fields in Hello packet is derived and used by the neighbor table with the same meaning. Distance $\mathrm{D}(\mathrm{i}, \mathrm{j})$ and the cost $\mathrm{Cj}$ are calculated using the above equations 2 and 3 respectively. Values of Device type DTj which is lower, Distance factor $\mathrm{D}(\mathrm{i}, \mathrm{j})$ with a shorter distance and higher remaining energy REj is used 
to compute the Communication cost $\mathrm{Cj}$ which is lower. With every new Hello packets received, the neighbour table constructor algorithm updates the neighbour table by computing the cost of communication.

\subsubsection{Routing table building Algorithm}

A single destination can have multiple records in Neighbor Table building algorithm. We can choose table entries with lowest communication cost using Routing Table building algorithm. This algorithm can help filter the table and select entries with lower cost for communication.

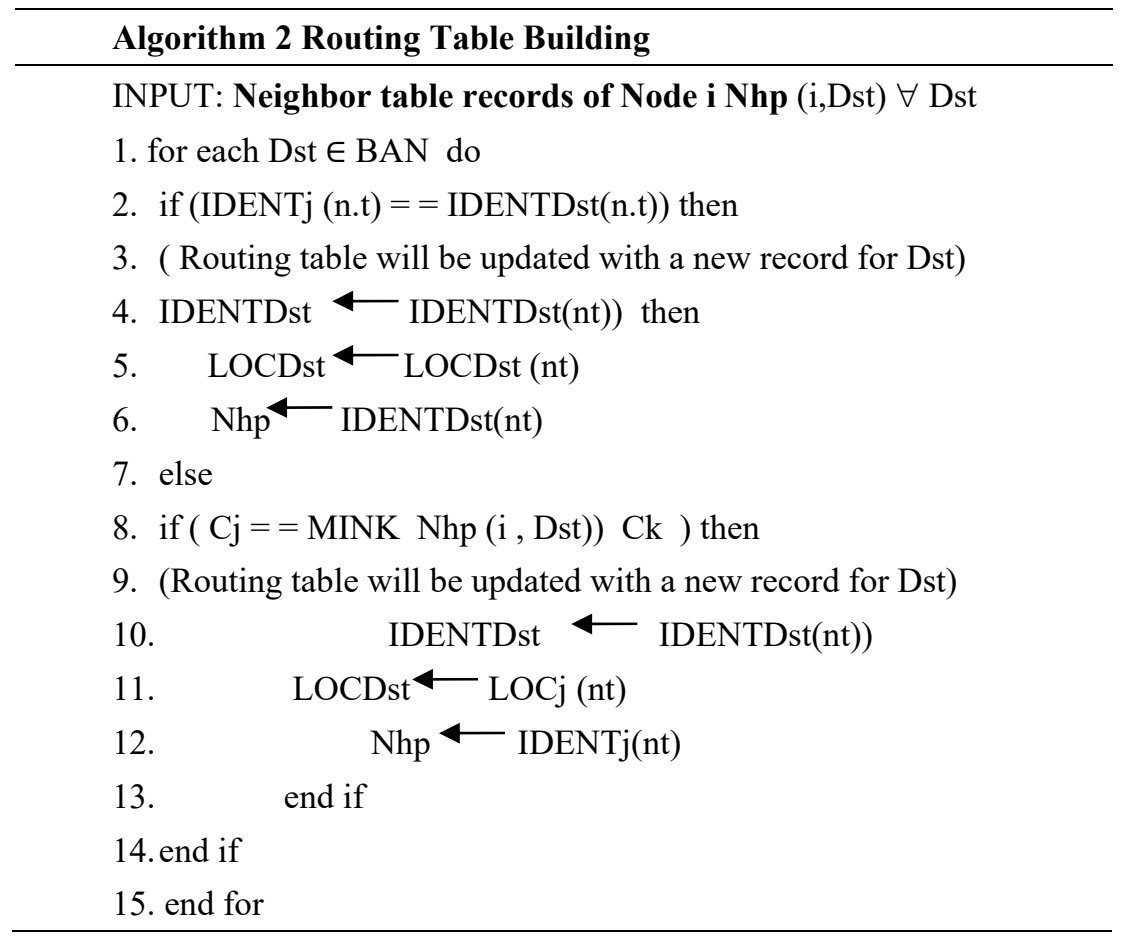

Figure 3 shows routing table structure of node $\mathrm{i}$ with the following fields. The field IDENTDst has the destination ID, destination location is available in LOCDst and Nhp has the next hop information. Algorithm 2 given below adds record to the destination Dst.

\begin{tabular}{|l|l|l|}
\hline IDENTDst & LOCDst & Nhp \\
\hline
\end{tabular}

Figure 3: Routing table Fields

Source and destination nodes if connected directly will result in the next hop Nhp being the destination. Else the algorithm will select the neighbor node $\mathrm{j}$ having the lower cost of communication $\mathrm{Cj}$ as the next hop node Nhp.

\subsection{Energy Model}

Residual energy of the sensor node is determined using the below equation 7. Here Sens is sensor node, Sens $_{R E}$ is the total remaining energy in the node. Sens ${ }_{A E}$ is the energy available in the sensor node. Sens $s_{C E}$ is the energy consumed by the sensor node .

$$
\text { Sens }_{R E}=\text { Sens }_{A E}-\text { Sens }_{C E}
$$

Sens ${ }_{C E}$ is computed using the number of bits transmitted and received in the sensor node. $B T_{x}$ is the number of transmitted bits and $B R_{x}$ is the number of received bits in the sensor node.Sen ${ }_{T E}$ andSen $n_{R E}$ is the total amount of transmitted and received energy as in equation 8.

$$
\operatorname{Sen}_{C E}=B T_{x} \times \operatorname{Sen}_{T E}+B R_{x} \times \operatorname{Sen}_{R E}
$$

$S e n_{E T}$ andSen $A E$ are the energy needed by the transmitter and amplifier respectively.D is the distance between the sender and the net forwarder node as shown in equation 9.

$$
\begin{aligned}
& \operatorname{Sen}_{T E}=\operatorname{Sen}_{E T}+\operatorname{Sen}_{A E} \times D \\
& \operatorname{Sen}_{R E}=\operatorname{Sen}_{E T}
\end{aligned}
$$




\section{Results and discussion}

The routing mechanism proposed is compared with the standard benchmark NEW-ATTEMPT protocol simulated in OMNET++ based Castalia simulator. Our routing mechanism is also compared with random routing where data packets are forwarded randomly to next hop hops without taking into consideration energy and related issues. Simulation has been done taking into consideration 3 possible events. In Event 1, the nodes are considered to be static or fixed without any changes in the topology of the network with the number of packets transmitted being fixed. In Event 2, the nodes are considered to be static and the number of packets transmitted is varied. In Event 3, the nodes have mobility i.e patients wearing the senor nodes are assumed to be moving at typical walking speed. Simulation uses the mechanism of random seeding and also the results obtained are average of repeating the simulation three times so that the results are smoother with very little or no variation.

Table 2: Simulation Parameters

\begin{tabular}{|c|c|c|}
\hline Simulation & \multicolumn{2}{|c|}{$\begin{array}{l}\text { Tool: OMNET ++ with Castalia. } \\
\text { Time: } 2000 \text { Seconds with } 5 \text { Seconds for initialization of node. } \\
\text { Results of Simulation: Average of } 3 \text { repetitions. }\end{array}$} \\
\hline MAC Layer & \multicolumn{2}{|c|}{ MAC IEEE 802.15 .4} \\
\hline Application & \multicolumn{2}{|c|}{ Event Driven } \\
\hline Packet Information & \multicolumn{2}{|c|}{ Size 32 Bytes with CBR Traffic } \\
\hline \multirow[t]{2}{*}{ Power related information } & Transmission Power used & $-10 \mathrm{dBm},-15 \mathrm{dBm},-25 \mathrm{dBm}$ \\
\hline & Reception power & $7 \mathrm{dBm}$ \\
\hline Transmission rate at Link layer & \multicolumn{2}{|c|}{$250 \mathrm{Kbps}$} \\
\hline Buffer size & \multicolumn{2}{|r|}{32 Packets } \\
\hline Number of nodes & \multicolumn{2}{|c|}{8 Numbers with one Sink Node and one Relay node. } \\
\hline
\end{tabular}

The benchmark parameters used to measure the performance of proposed protocol and compare with other similar protocols are Energy consumption by nodes, Overall traffic in the network, using nodes between the source and destination to forward the packets, buffer overflow at the MAC layer and Transmissions which are successful.

NEW-ATTEMPT creates energy drain due to cost function which considers only remaining energy and distance to the sink. This results in increase in the number of packet failures and retransmission with additional consumption of energy. This will reduce the life time of network. The cost function in our protocol EER-W considers multiple network parameters into account. This can increase the data transmission and throughput reducing energy consumption and improvement in network lifetime.

EER-W maintains packet delivery ratio which is steady compared to NEW-ATTEMPT and random routing. Also early channel saturation due to overhead of control packets was observed in other comparison protocols.

The results obtained for the above mentioned events with parameters considered have been discussed below.

\subsection{Event 1: Fixed Scenario}

In the first possible event, BAN nodes send $1 \mathrm{~K}$ packets to relay nodes or sink node. The nodes have been deployed as shown in Figure 4. Among all the BAN nodes, B1 is close to the sink node and relay node. This will result in Node B1 being frequently used to forward the packets of other nodes to sink node.

This will have an impact on the energy levels of node B1 with significant consumption .Node B1 also experiences huge congestion. The proposed routing mechanism overcomes the above mentioned issues by selecting appropriate next hop nodes with the lowest communication cost .The proposed routing mechanism is also optimized for energy conservation by avoiding unnecessary transmission of packets.

\subsubsection{Packets forwarded by intermediate nodes}

The below Figure 5 shows how the packets have been routed using nodes which are intermediate between source and destination. From the below Figure 5, we can see that our EER-W protocol use intermediate nodes to forward 295 packets of data using transmission power of $-25 \mathrm{dBm}$. When the transmission power is $15 \mathrm{dBm}$ and $10 \mathrm{dBm}$ the destination nodes are within the range of source nodes. As a result of this, no nodes between source and destination is used as intermediate to transmit packets. 


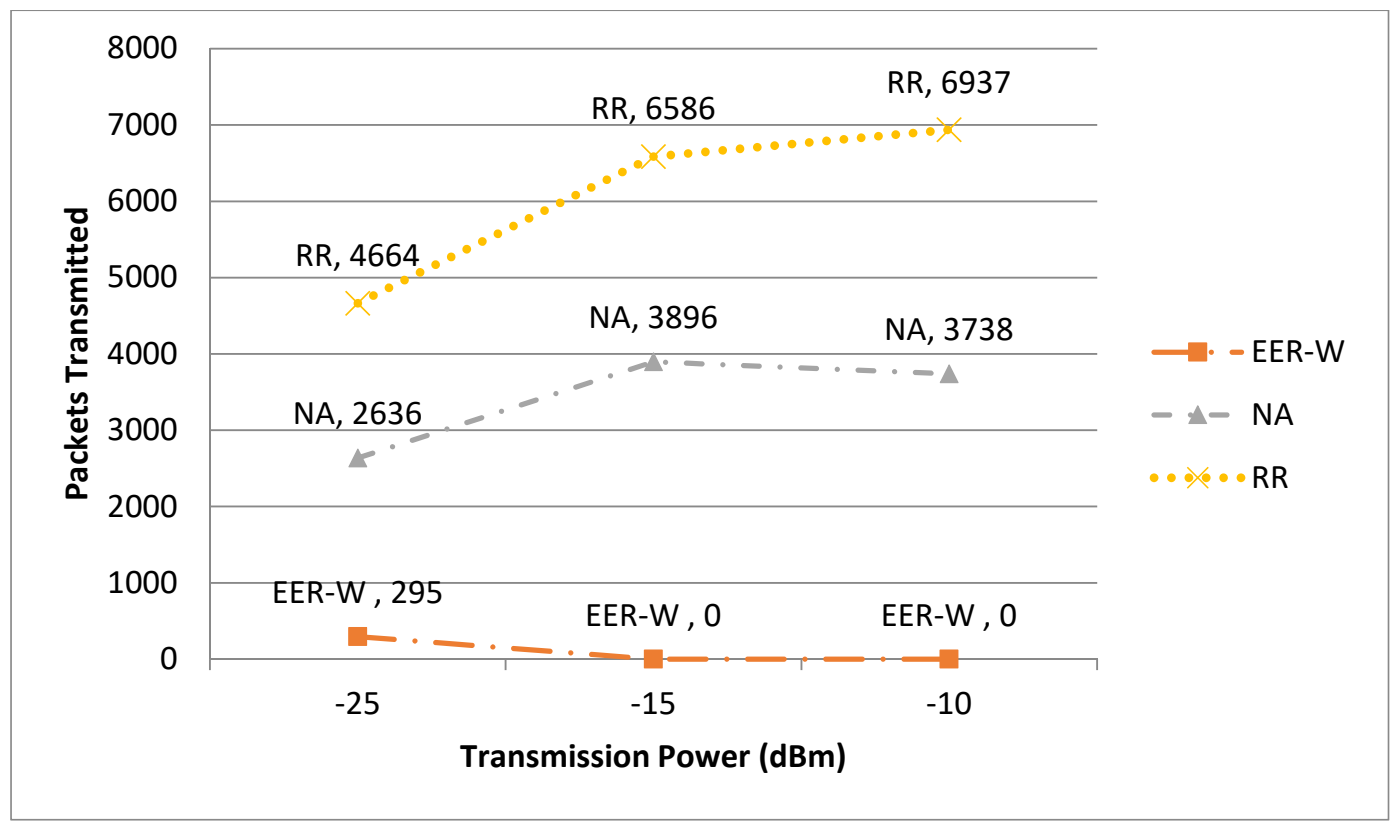

Figure 5: Packets Forwarded by intermediate nodes

When compared to this, the NEW-ATTEMPT protocol uses intermediate nodes to forward 2636, 3896 and 3738 packets for transmission power of $-25 \mathrm{dBm},-15 \mathrm{dBm}$ and $-10 \mathrm{dBm}$ respectively.

\subsubsection{Network traffic and Energy consumption}

When no routing mechanism is used and packets are forwarded using random routing, 4664, 6586 and 6937 packets are transmitted using intermediate nodes for transmission power of $-25 \mathrm{dBm},-15 \mathrm{dBm}$ and $-10 \mathrm{dBm}$ respectively.

Our EER-W protocol is energy efficient because of less number of Hello packets and data packet transmissions. This also reduces the overall network traffic. This has been illustrated in Figure 5 and 6 . The below Figure 5 shows the traffic reduced in EER-W compared to NEW-ATTEMPT and Random Routing. Also we can observe that the traffic load in the network has increased by $100 \%$ when compared to EER-W for all the three transmit powers.

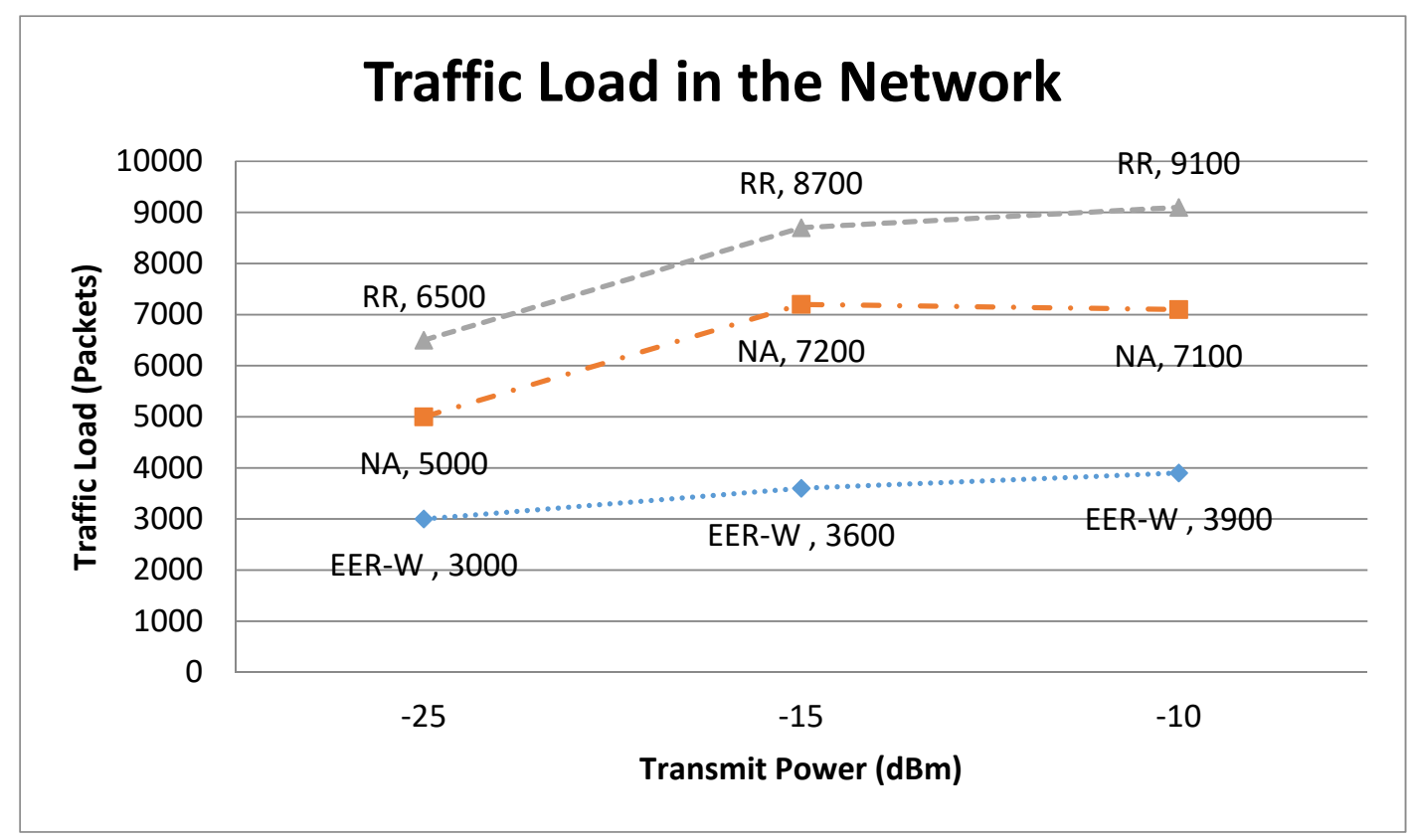

Figure 6: Traffic Load in the Network 


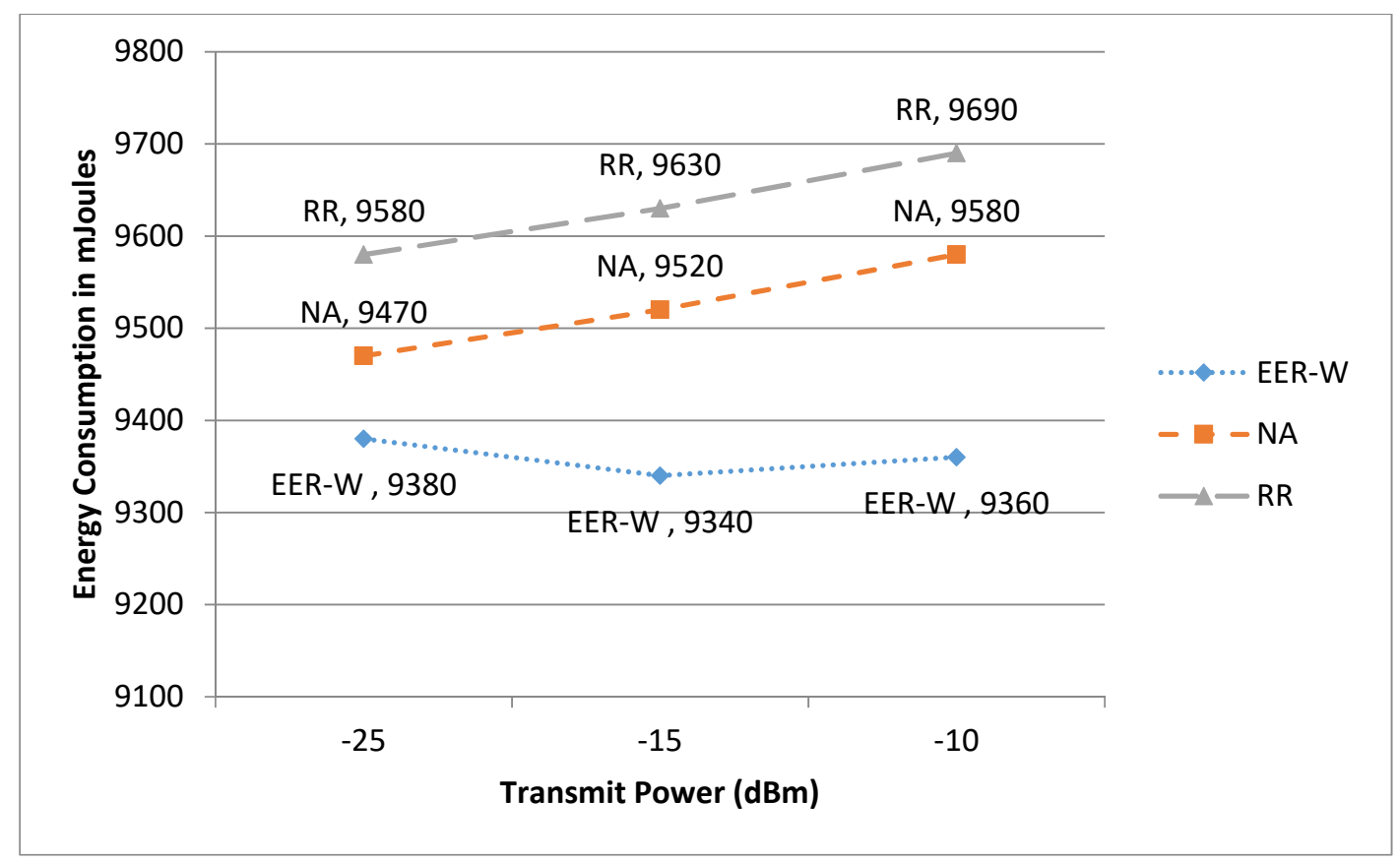

Figure 7: Cumulative Energy Consumption

EER-WRouting Mechanism consumes $9491 \mathrm{~mJ}, 9224 \mathrm{~mJ}$ and $9483 \mathrm{~mJ}$ when the transmit powers are $-25 \mathrm{dBm}$, $-15 \mathrm{dBm}$, and $-10 \mathrm{dBm}$ respectively. The benchmark protocol NEW-ATTEMPT for the same energy levels consume $9383 \mathrm{~mJ}, 9445 \mathrm{~mJ}$ and $9497 \mathrm{~mJ}$ energies respectively. Our protocol saves significant amount of energy. 5.1.3 Packet drop due to buffer overflow

Packet drop due to buffer overflow has been represented in Figure 8 . When the transmission power is -25 dBm, packets dropped by EER-W, NEW-ATTEMPT is negligible as shown in Figure 8. For -15 dBm EER-NT, NEW-ATTEMPT and RR drops 664, 985 and 1876 packets respectively. For -10 dBm EER-NT, NEWATTEMPT and RR drop 757, 932, and 1904 packets respectively. From the below figure we can see that the packet drop rate in EER-W is very less compared to the other approaches for different levels of powers during transmission.

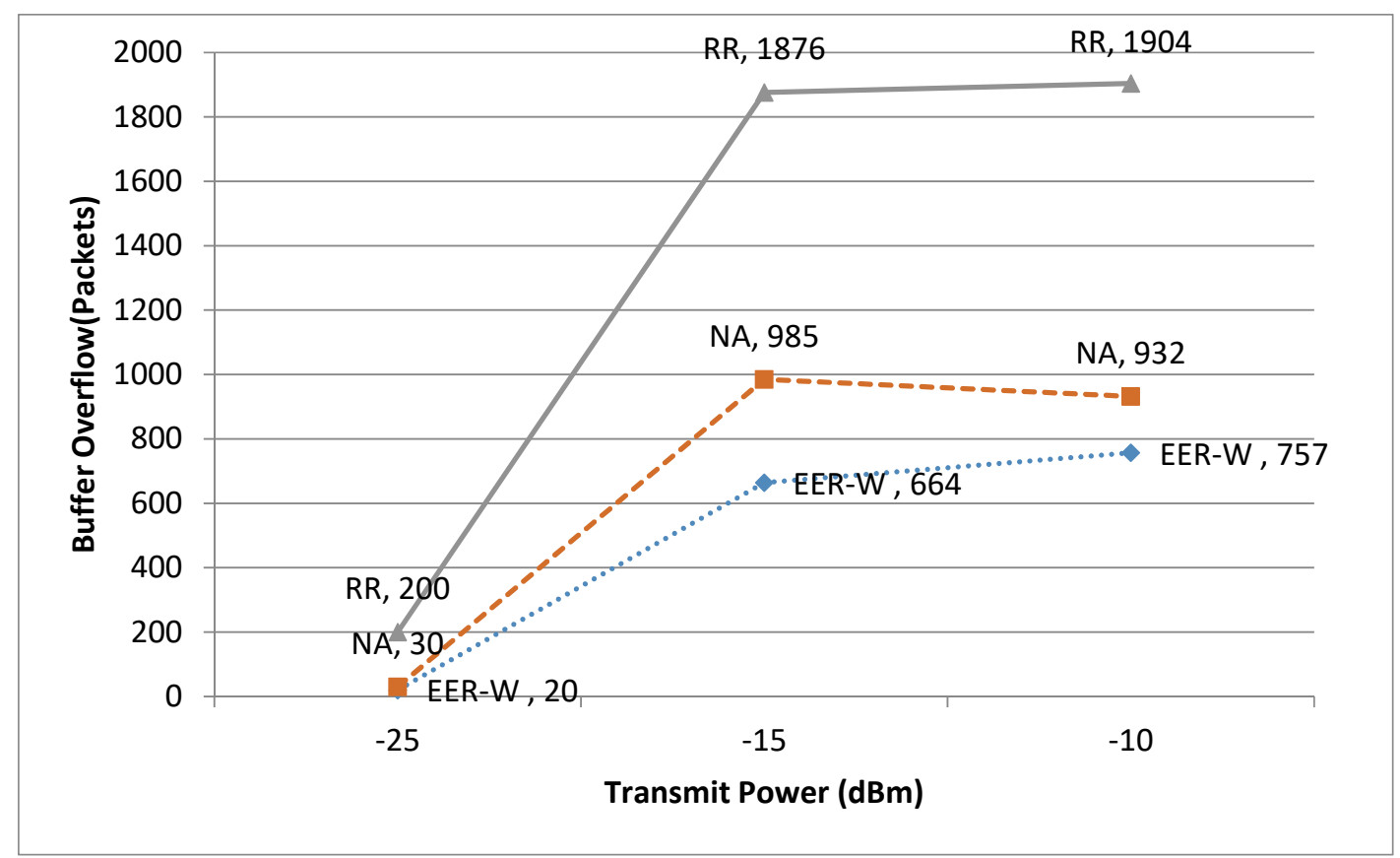

Figure 8: Packets dropped due to buffer overflow 


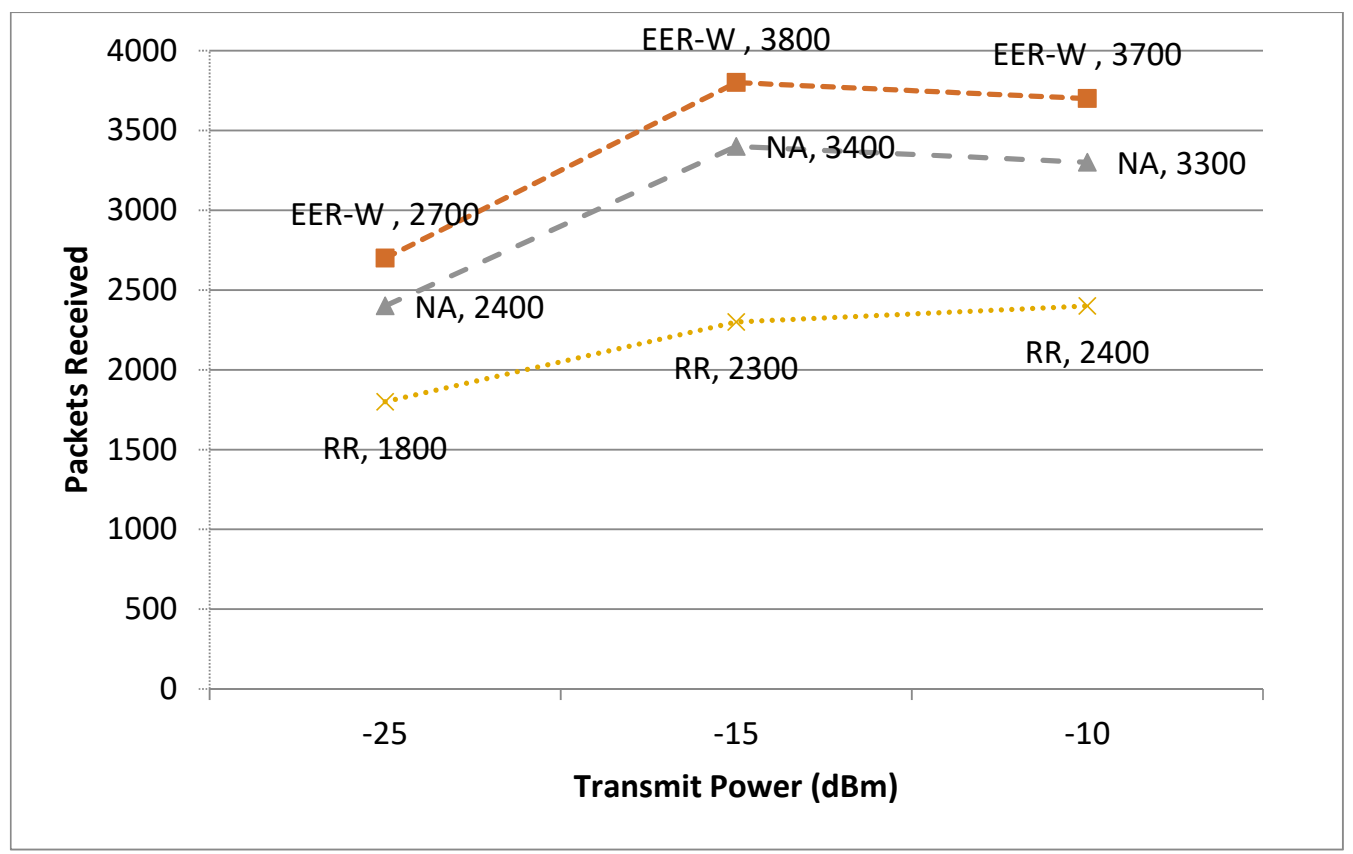

Figure 9: Throughput and Transmit powers

EER-W protocol reduces the network traffic and this reduction results in more chances of data packet successfully reaching the destination. When compared with NEW-ATTEMPT and RR, we see EER-W delivers more packets as shown in Figure 9.

\subsection{Event 2: Mobile Scenario}

Here the patient is assumed to be mobile with walking speed of 1 meter per second. Observations were made for the following 1.Network Throughput 2. Number of packets forwarded by intermediary node.3. Buffer Overflow 4.End to End delay between source and destination. The transmission power that was used was $25 \mathrm{dBm}$.It was observed that EER-NT provides better results than NEW-ATTEMPT and RR for mobile scenario. Throughput for EER-Wwas $75 \%-85 \%$ compared to $60 \%-65 \%$ for NEW-ATTEMPT and $45 \%$ to $50 \%$ for RR.

\subsubsection{Throughput}

Throughput measures the number of packets arriving at the destination successfully. As shown in Figure 10, EER-W has a throughput between $66 \%$ to $75 \%$ for low transmission power of $-25 \mathrm{dBm}$. NEW-ATTEMPT protocol provides throughput between $62 \%$ to $68 \%$ and RR has throughput of $46 \%$ to $51 \%$.

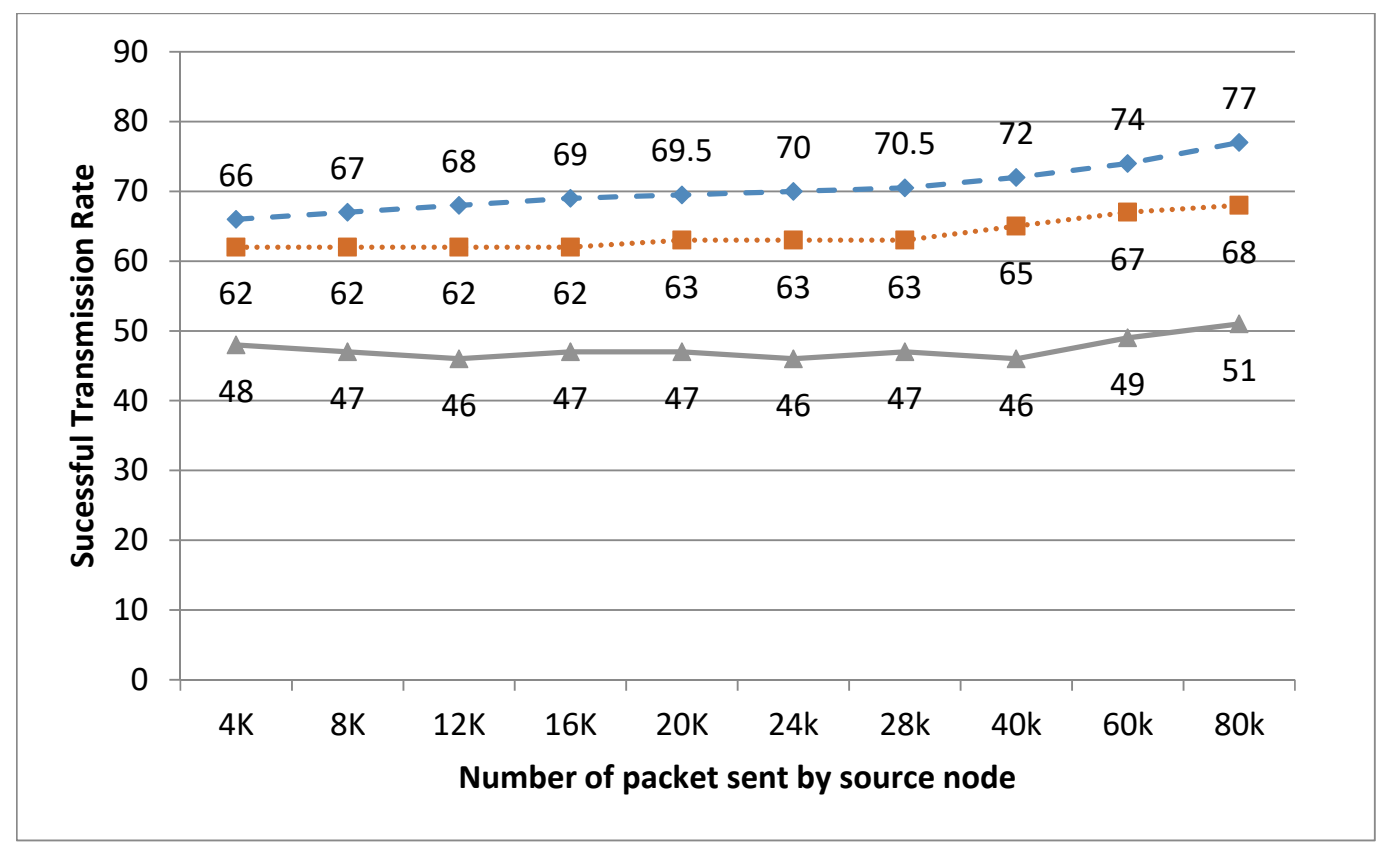

Figure 10: Throughput for Offered load when for $-25 \mathrm{dBm}$ 


\subsubsection{Packets forwarded by intermediate nodes vs Offered traffic}

When the transmission power is $-25 \mathrm{dBm}$, EER-W protocol transmits 345 to 7735 packets using intermediate nodes. For the same energy level NEW-ATTEMPT protocols forward 2600 packets to 55,500 packets using intermediate node. RR protocol forwards 4500 to 97,800 packets respectively as shown in Figure 11.

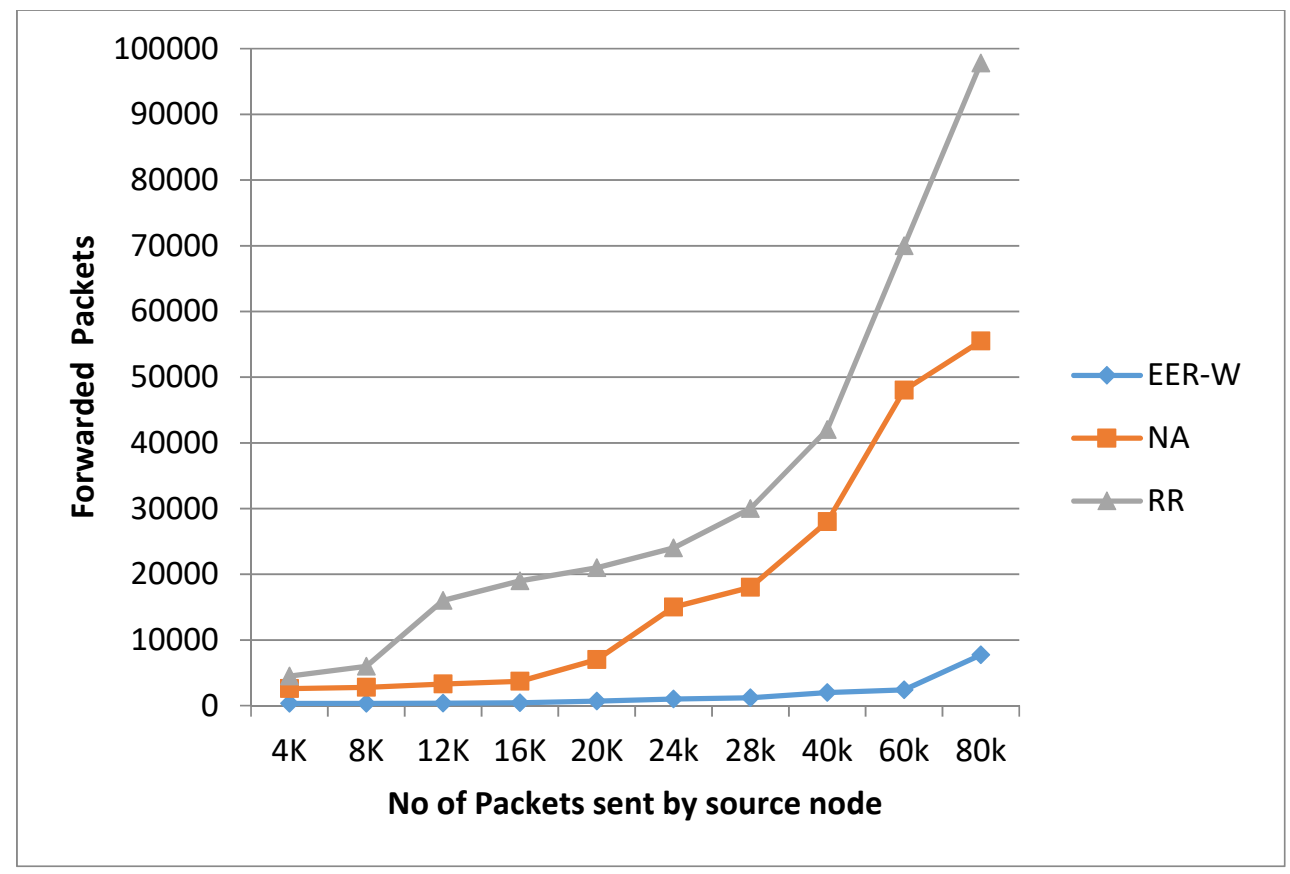

Figure 11: Packets forwarded by intermediate nodes vs Offered load when transmit power is $-25 \mathrm{dBm}$

The improvement in overall performance is because of EER-W protocol chooses the next hop node based in the low communication cost. Hello packets are broadcasted only if needed. When the transmission power is higher i.e. for $-15 \mathrm{dBm}$ and $-10 \mathrm{dBm}$.

EER-Wprotocol has been designed to send the data packet directly to destination if it is in range. When the transmission power is less than $-15 \mathrm{dBm}$ EER-W protocol uses intermediatory nodes since destination nodes are not in range.

If not intermediatory nodes are taken into consideration .Other similar benchmark protocols like NEWATTEMPT and RR use the mechanism of forwarding the packets to the nearest nodes which are upstream nodes.

\subsubsection{Network Traffic}

The improvised strategies used in EER-W results in reduction of the number of Hello packets and Data packets which are forwarded by intermediate nodes. This reduces the overall network traffic compared to other benchmark methods.

Figure 11 compares the performance of EER-W with NEW-ATTEMPT and RR in terms of network traffic load for different transmission powers. It can be observed that EER-W generates less network traffic. 


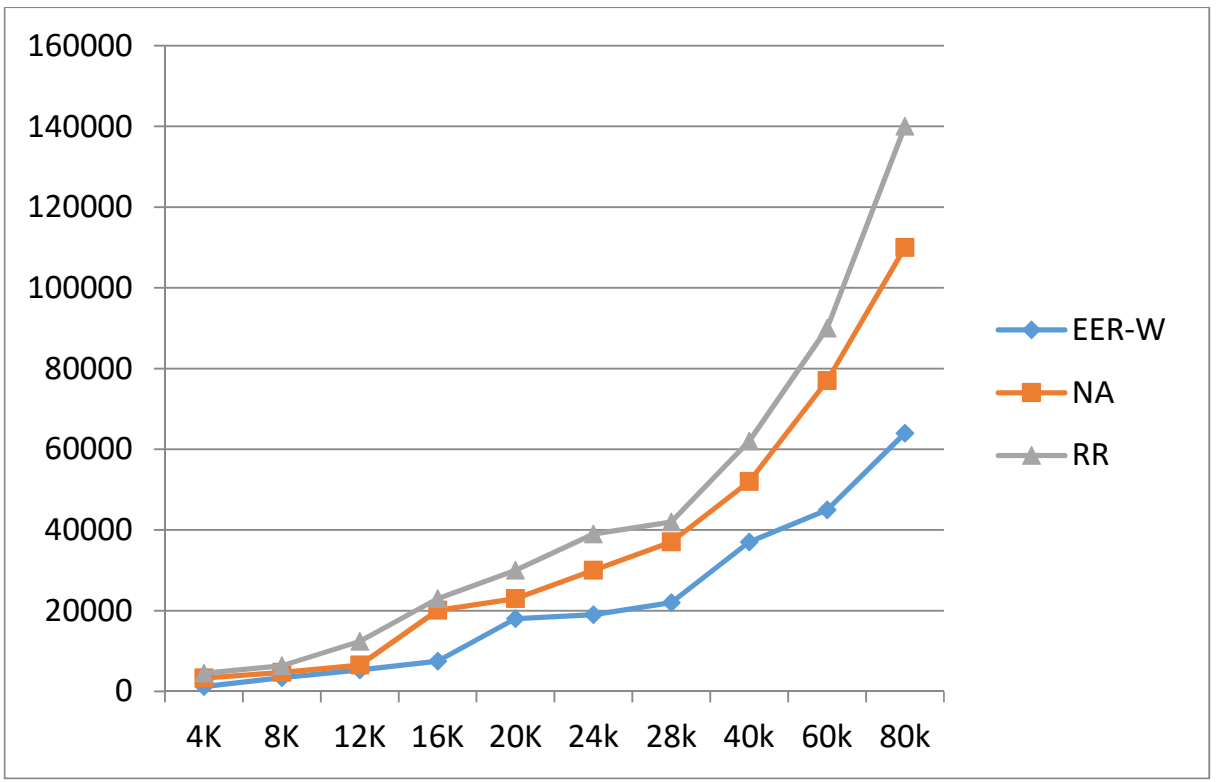

Figure 12: Traffic Load in Network vs Number of packets for $-25 \mathrm{dBm}$

From Figure 12 it can be observed that EER-W reduces the network traffic significantly for all transmission powers. At low transmission power more data packets go through intermediate nodes.

\section{Conclusion}

In this paper, we have proposed an energy efficient routing protocol EER-W which improves the throughput and lifetime of the network. We introduced efficient mechanisms for reducing the number of control packets and load in the network. A path cost computation function based on sensor node type, residual energy and distance to the sink was used for the selection of the next-hop to transmit the data packets. We performed extensive simulation on OMNET++ based Castalia simulator by varying number of packets and transmission power .The performance of the protocol EER-W was analyzed for the network considering the static and mobile scenarios for various metrics like energy consumption, throughput and network lifetime.

The experimental results show EER-W consumes less energy with high throughput reduced packet loss and improved network lifetime compared to the protocol NEW-ATTEMPT and random routing.

\section{References}

[1] T. M. Dall, P. D. Gallo, R. Chakrabarti, T. West, A. P. Semilla, and M. V. Storm, "The care span: An aging population and growing disease burden will require a large and specialized health care workforce by 2025," Health Aff., vol. 32, no. 11, pp. 2013-2020, 2013.

[2] D. N. Mendelson and W. B. Schwartz, "The effects of aging and population growth on health care costs," Health Aff., vol. 12, no. 1, pp. $119-125,1993$.

[3] A. Darwish and A. E. Hassanien, "Wearable and implantable wireless sensor network solutions for healthcare monitoring," Sensors, vol. 11, no. 6, pp. 5561-5595, 2011.

[4] S. Macwan, N. Gondaliya, and N. Raja, "Survey on Wireless Body Area Network," vol. 5, no. 2, pp. 107-110, 2016.

[5] S. Ullahet al., "A comprehensive survey of wireless body area networks on PHY, MAC, and network layers solutions," J. Med. Syst., vol. 36, no. 3, pp. 1065-1094, 2012.

[6] A. Sundar Raj and M. Chinnadurai, "Energy efficient routing algorithm in wireless body area networks for smart wearable patches," Comput. Commun., vol. 153, no. February, pp. 85-94, 2020.

[7] G. Yang, X. W. Wu, Y. Li, and Q. Ye, "Energy efficient protocol for routing and scheduling in wireless body area networks," Wirel. Networks, vol. 26, no. 2, pp. 1265-1273, 2020.

[8] K. N. Qureshi, F. Bashir, and A. H. Abdullah, "An energy and link aware next node selection protocol for body area networks," Int. Conf. Inf. Netw., vol. 2018-Janua, pp. 721-726, 2018.

[9] S. Ahmed et al., "Co-LAEEBA: Cooperative link aware and energy efficient protocol for wireless body area networks," Comput. Human Behav., vol. 51, pp. 1205-1215, 2015.

[10] B. Abidi, A. Jilbab, and E. H. Mohamed, “An energy efficiency routing protocol for wireless body area networks," J. Med. Eng. Technol., vol. 42, no. 4, pp. 290-297, 2018.

[11] M. Roy, C. Chowdhury, and N. Aslam, "Designing an energy efficient WBAN routing protocol," 2017 9th Int. Conf. Commun. Syst. Networks, COMSNETS 2017, pp. 298-305, 2017.

[12] O. Smail, A. Kerrar, Y. Zetili, and B. Cousin, "ESR: Energy aware and stable routing protocol for WBAN networks," 2016 Int. Wirel. Commun. Mob. Comput. Conf. IWCMC 2016, pp. 452-457, 2016.

[13] V. Navya and P. Deepalakshmi, "Energy efficient routing for critical physiological parameters in wireless body area networks under mobile emergency scenarios," Comput. Electr. Eng., vol. 72, pp. 512-525, 2018.

[14] N. Javaid, A. Ahmad, Q. Nadeem, M. Imran, and N. Haider, "iM-SIMPLE: iMproved stable increased-throughput multi-hop link efficient routing protocol for Wireless Body Area Networks," Comput. Human Behav., vol. 51, pp. 1003-1011, 2015.

[15] N. Kaur and S. Singh, "Optimized cost effective and energy efficient routing protocol for wireless body area networks," Ad Hoc Networks, vol. 61, pp. 65-84, 2017. 
[16] Y. Qu, G. Zheng, H. Wu, B. Ji, and H. Ma, "sensors An Energy-E fficient Routing Protocol for Reliable data transmission in wireless body area networks," 2019.

[17] M. Anwar et al., "Green communication for wireless body area networks: Energy aware link efficient routing approach," Sensors (Switzerland), vol. 18, no. 10, 2018.

[18] H. Fouad, "Continuous Health-monitoring for early Detection of Patient by Web Telemedicine System," pp. 76-83, 2001.

[19] B. Latré, B. Braem, I. Moerman, C. Blondia, and P. Demeester, "A survey on wireless body area networks," Wirel. Networks, vol. 17, no. 1 , pp. $1-18,2011$

[20] M. Ghamari, B. Janko, R. S. Sherratt, W. Harwin, R. Piechockic, and C. Soltanpur, "A survey on wireless body area networks for ehealthcare systems in residential environments," Sensors (Switzerland), vol. 16, no. 6, pp. 1-33, 2016.

[21] L. Hughes, X. Wang, and T. Chen, A review of protocol implementations and energy efficient cross-layer design for wireless body area networks, vol. 12, no. 11.2012.

[22] Oguchi, "Content Transfer and Supporting Technologies in a Home Environment over Next Generation Convergence Home Network From Vital Information Transfer to Broadband Content Transfer," Int. J. Digit. Content Technol. its Appl., vol. 3, no. 3, 2009.

[23] S. Singh, S. Negi, A. Uniyal, and S. K. Verma, "Modified new-Attempt routing protocol for wireless body area network," Proc. - 2016 Int. Conf. Adv. Comput. Commun. Autom. (Fall), ICACCA 2016, 2016.

[24] Chakrabarti, S. (2000): Data mining for hypertext: A tutorial survey. SIGKDD explorations, 1(2), pp. 1-11. 